\title{
Regressangst der Ärzte schadet Patienten
}

\author{
Die Arzneimittelbudgetierung mit der daraus folgenden Angst vor Regress- \\ forderungen verunsichert die Ärzte. Am Quartalsende unterbleiben dann \\ gelegentlich auch eigentlich notwendige Verordnungen - zum Schaden von \\ Patienten, die nicht dem „Verordnungsdurchschnitt“ angehören.
}

— In seiner Sendung vom 25.1.2012 hat das ARD-Verbrauchermagazin „Plusminus" anhand zweier Beispiele die Gefahren von Regressen sehr deutlich gemacht. Berichtet wurde über den kleinen Nick, der wegen der verschiedener notwendiger Darmoperationen künstlich ernährt werden muss und täglich Infusionen braucht. Die 53 Rezepte pro Monat für diese Infusionen wollte die zuständige Kinderärztin aber nicht mehr auf ihre Kappe nehmen: Sie stellte keine Rezepte mehr aus.

Solches Sparen sei von KV-Seite zwar nicht gewünscht, erklärte Dr. Peter Potthoff, Vorstandsvorsitzender der hier zuständigen KV Nordrhein. Aber die KV kenne das Problem, dass Ärzte versuchten, Regressen zu entgehen, indem sie zum Ende des Quartals Verordnungen aussetzten.

\section{Angst nicht ganz unbegründet}

Die Angst der Ärzte ist nicht unbegründet, wie der Fall von Hausarzt Odilo Schnabel aus Zaberfeld in der Nähe von Heilbronn zeigt. Schnabel gelte als einer der teuersten Ärzte Baden-Württembergs, hieß es. In fünf Jahren hat er eine Regresssumme von 417000 Euro angesammelt, die er kaum aus seinem Privatvermögen stemmen kann.

„Mein Verordnungsverhalten werde ich trotzdem nicht ändern", sagte Schnabel. Denn seine Patienten auf dem Land seien auf ihn angewiesen. Vor allem viele Senioren würden den Weg zum Facharzt nicht schaffen. Schnabel hat deshalb Widerspruch gegen die Regresse eingelegt.

\section{Gesetz bannt Regresse nicht}

Im Bundesgesundheitsministerium (BMG) scheint die Angst, die die Ärzte umtreibt und die Versorgungslücke, die sich auftut, noch nicht angekommen zu sein. Denn die Anfrage von „Plusminus“ beim BMG habe gezeigt, dass man dort „nicht einmal wisse, wie viele Ärzte mit Regressforderungen konfrontiert sind“.

Immerhin wolle man die extremsten Auswüchse durch Gesetzesänderungen abmildern, hieß es. Ob das Versorgungsstrukturgesetz dies mit seinem Prinzip „Beratung vor Regres“" schafft,

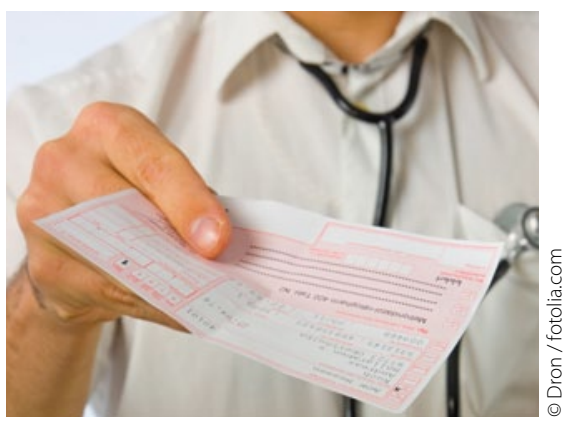

Viele Ärzte arbeiten unter der ständigen Angst vor Regressansprüchen.

ist allerdings fraglich. Dadurch haben die Ärzte zwar unter anderem einen Anspruch auf Beratung, wenn sie das erste Mal die Richtgrößen überschreiten, die eigentlichen Regressforderungen bannt das Gesetz aber nicht.

\section{Zulassungsgremien bleiben bei Einigung außen vor}

\section{Gebot bestimmt den Praxiswert}

\section{Wenn sich die beteiligten Ärzte über den Wert einer Praxis einig sind, dürfen die Zulassungsgremien nach einer Entscheidung des Bundessozialgerichts (BSG) nicht noch selbst einen Praxiswert ermitteln.}

\begin{abstract}
— Im Streitfall hatte eine Psychotherapeutin in Baden-Württemberg sich mit den interessierten Kollegen auf einen Verkehrswert von 40000 Euro geeinigt. Trotzdem holte der Berufungsausschuss ein eigenes Gutachten ein, das einen Wert von 35000 Euro ergab. Auch das gefiel dem Ausschuss nicht. Ein weiteres Gutachten ergab einen immateriellen Praxiswert von Null. So verblieb nur noch ein Sachwert von 2940 Euro. Das LSG Baden-Württemberg sorgte für Aufsehen mit der Aussage, der Ausschuss sei grundsätzlich berechtigt gewesen, einen Praxiswert festzusetzen.
\end{abstract}

Dem trat das BSG nun klar entgegen. Hat sich der abgebende Arzt mit einem oder mehreren Interessenten auf einen Praxiswert geeinigt, um so die Nachfolgeentscheidung $\mathrm{zu}$ beschleunigen, haben sich die Zulassungsgremien nicht mehr einzumischen. Nur wenn es verschieden hohe Gebote gibt, müssen die Zulassungsgremien einen Wert festsetzen. Dieser darf aber nicht unter dem niedrigsten Gebot liegen, weil sich die Interessenten zumindest in dieser Höhe einig sind.

MWO =

- Az.: BSG: B 6 KA 39/10 R; Vorinstanz LSG: L 5 KA 1323/09 